\title{
PREVALENCIA DE FACTORES DE RIESGO CARDIOVASCULAR EN PACIENTES HOSPITALIZADOS EN UN HOSPITAL DE LIMA
}

\author{
PREVALENCE OF CARDIOVASCULAR RISK FACTORS IN HOSPITALIZED PATIENTS IN A LIMA HOSPITAL
}

Karla X. Zuni-Chavez',2, Bryan Emilio More-Sandoval', Carlos Daniel Fernández-Vargas',

Bill Bryan García-Fuentes', Julio Manuel Ruiz-Olano³, Vanessa Karin Pérez-Rodriguez ${ }^{3}$

\begin{abstract}
RESUMEN
Objetivo: Determinar los factores asociados a riesgo cardiovascular en pacientes del Departamento de Medicina del Hospital Sergio E. Bernales (HSEB). Métodos: Estudio transversal, descriptivo y observacional, incluyo 83 pacientes elegidos probabilísticamente de una población de 105 hospitalizados del Departamento de Medicina del HSEB. En el análisis e interpretación de los datos se utilizaron gráficos y tablas de frecuencias relativas y absolutas. Resultados: El 53\% fueron mujeres y la edad promedio fue 54,5 años. La frecuencia de factores de riesgo principales fue: El sobrepeso $30,1 \%$, la obesidad 13,3\%, el Índice Ci/Ca muy alto en mujeres $54,2 \%$, antecedente familiar de IAM 38,5\% e HTA 34,6\%, DM2 65,5\%, Colesterol Alto 34,4\%, HTA 21.7\%, según su tipo se tuvo que el $73.9 \%$ estaban controladas; y al clasificarla, fueron optimas el $21,7 \%$ y Normal-Alta 34,8 , asimismo se observó también sedentarismo $50 \%$, consumo carbohidratos $56.6 \%$, lípidos $32.5 \%$, comida rápida $44,6 \%$ y como grado de Ansiedad menor un $54.2 \%$ y mayor el $32,5 \%$. Conclusión: Un mayor riesgo de evento coronario afecta más a las mujeres mayores de 50 años que en los varones, teniendo como factores de riesgo cardiovascular principales la dieta, el sedentarismo, el sobrepeso, las presiones normal- alta, la ansiedad menor, y comorbilidades asociadas como la diabetes y colesterol alto.
\end{abstract}

Palabras clave: Mujer; Sedentarismo; Sobrepeso; Ansiedad. (fuente: DeCS BIREME)

\begin{abstract}
Objective: To determine the factors associated with cardiovascular risk in patients of the HSEB Department of Medicine. Methods: Prospective, cross-sectional, descriptive and observational study, included 83 probabilistically chosen patients from a universe of 105 hospitalized from the HSEB Department of Medicine. For the calculation, OpenEpi version 3 and Microsoft Excel 2010 were used and in the analysis and interpretation of the data, graphs and tables of relative and absolute frequencies were used. Results: $53 \%$ were women and the average age was 54.5 years. The frequency of main risk factors was: Overweight $30.1 \%$, obesity $13.3 \%$, the $\mathrm{Ci}$ / Ca index very high in women $54.2 \%$, family history of AMI $38.5 \%$ and HTA $34.6 \%$, DM2 $65.5 \%$, High Cholesterol $34.4 \%$, HTA $21.7 \%$ of these $73.9 \%$ were controlled, classified and were optimal $21.7 \%$ and Normal-High 34.8 , also sedentary lifestyle $50 \%$, carbohydrate consumption $56.6 \%$, lipids $32.5 \%$, fast food $44.6 \%$ and as a degree of Anxiety less than $54.2 \%$ and greater than $32.5 \%$. Conclusion: There is a higher risk of a coronary event more in women over 50 years of age than in men, having as main cardiovascular risk factors diet, sedentary lifestyle, overweight, normal-high pressures, minor anxiety, and comorbidities Associated such as diabetes and high cholesterol.
\end{abstract}

Key words: Women; Sedentary; Overweight; Anxiety. (source: MeSH NLM)

1 Facultad de Medicina Humana, Universidad Alas Peruanas, Lima-Perú.

2 Sociedad Científica y Académica de Estudiantes de medicina de la Universidad Alas Peruanas, Lima-Perú.

${ }^{3}$ Departamento de Epidemiologia del Hospital Sergio E. Bernales, Lima-Perú.

Citar como: Karla X. Zuni-Chavez, Bryan Emilio More-Sandoval, Carlos Daniel Fernández-Vargas, Bill Bryan García-Fuentes, Julio Manuel Ruiz-Olano, Vanessa Karin Pérez-Rodriguez. Prevalencia de factores de riesgo cardiovascular en pacientes hospitalizados en un hospital de Lima. Rev. Fac. Med. Hum. Octubre 2019; 19(4):68-73. DOI 10.25176/RFMH.v19i4.2343

Artículo publicado por la Revista de la Facultad de Medicina Humana de la Universidad Ricardo Palma. Es un artículo de acceso abierto, distribuído bajo los términos de la Licencia Creative Commons: Creative Commons Attribution 4.0 International, CC BY 4.0 (https://creativecommons.org/licenses/by/4.0/), que permite el uso no comercial, distribución y reproducción en cualquier medio, siempre que la obra original sea debidamente citada. Para uso comercial, por favor póngase en contacto con revista.medicina@urp.pe 


\section{INTRODUCCIÓN}

Las enfermedades cardiovasculares (ECV) son un trastorno crónico que se desarrolla de forma insidiosa, progresando incluso cuando no aparecen síntomas y/o signos, elevando más el grado de discapacidad al momento del diagnóstico', se estima que en las próximas décadas la pérdida total de años de vida ajustados por discapacidad (AVAD) aumentaría de 85 millones en 1990 a 150 millones en $2020^{2,3}$ y, por tanto, continuaría siendo la causa más importante de pérdida de productividad. La ECV está fuertemente relacionada con el estilo de vida, especialmente con el consumo de tabaco, los hábitos alimentarios poco saludables, la inactividad física y el estrés psicosocial ${ }^{4,5}$. Según la OMS, con cambios adecuados del estilo de vida se podría prevenir más de tres cuartas partes de la mortalidad cardiovascular ${ }^{6}$ ya que continua siendo un reto importante para la población, los actores políticos y los profesionales de la salud, quienes con acciones coordinadas, poblacionales e individuales busquen erradicar, eliminar o minimizar el impacto de las ECV y la discapacidad asociada a ellas ${ }^{7,8}$.

El estudio nos proporcionara medidas preventivas y rehabilitadoras a la población sobre las consecuencias que pudieran desarrollar, debido a sus hábitos de vida perjudiciales que, poco a poco disminuyen su esperanza de vida, por tanto, el presente trabajo tiene como objetivo principal describir la frecuencia de los factores de riesgo cardiovascular en pacientes del Departamento de Medicina del Hospital Sergio E. Bernales (HSEB).

\section{MÉTODOS}

Se incluyó a 83 pacientes elegidos probabilísticamente de una población de 105 pacientes hospitalizados del servicio de Medicina del HSEB durante setiembre del 2016, se utilizó la fórmula estadística de proporciones para población finita con un nivel de confianza del $95 \%$, un error relativo de $5 \%$ y una frecuencia esperada del 50\%; utilizando la calculadora de código abierto del OpenEpi, seleccionándose a los participantes de la lista de hospitalizados en el mes mencionado por un tiempo de dos semanas.

Se realizo una metodología cuantitativa, con un diseño prospectivo, transversal, descriptivo $y$ observacional, con la aplicación de una ficha de recolección de datos de elaboración propia, validada por expertos en el tema. Para determinar los factores de riesgo cardiovascular se capacito previamente a los encuestadores para la recolección de los datos que incluyó: diagnóstico de Hipertensión Arterial (HTA) mediante el promedio de al menos 2 tomas de presión arterial separadas por 5 minutos en posición sedente y en reposo de 15 minutos previos a la toma ${ }^{9,10}$, utilizándose esfigmomanómetros manuales marca Riester, modelo minimus ${ }^{\circledR}$, clasificando a los pacientes mediante la guía Europea de cardiología (2012) ${ }^{11}$ para hipertensión arterial. Para la medición de la talla se usó un tallímetro que sigue las recomendaciones del Centro Nacional de Alimentación y Nutrición (CENAN)12; para la determinación del peso fue mediante una balanza digital electrónica calibrada (Seca Alpha, GmbH \& Co., Igni, France; rango 0,1$150 \mathrm{~kg}$, precisión $100 \mathrm{~g}$; determinación del índice cintura/cadera (ICC), la medición de la cintura se realizó tomando el punto medio entre la arcada costal inferior y cresta iliaca superior a nivel de la línea axilar anterior, y la cadera, en la parte más ancha al nivel bi-trocantérico; el índice de masa corporal (IMC) y el índice cintura cadera (ICC) fueron analizadas según lo establecido por la Organización Mundial de la Salud $(\mathrm{OMS})^{6}$ y para determinar el nivel de ansiedad se utilizó el test de Hamilton ${ }^{13,14}$.

\section{Procedimientos}

Se solicito una cita con el Director Médico del HSEB donde se le informo de los objetivos y justificación de la presente investigación, una vez aprobada la solicitud, la dirección médica se hizo responsable de enviar el documento pertinente al jefe de área del Departamento de Medicina informando sobre los detalles del desarrollo del estudio y a la vez comprometiéndose a brindar las facilidades necesarias al momento de aplicar las encuestas.

Una vez realizadas las coordinaciones previas y teniendo la autorización de la institución, se procedió a aplicar la encuesta a los pacientes del Departamento de Medicina, el encuestador informo de manera verbal y escrita al paciente de los objetivos del estudio, así como también de la importancia y beneficios de su participación en el desarrollo de la investigación. Una vez aceptada su participación se les proporciono un biombo para mayor privacidad y confidencialidad del proceso, acompañado por el encuestados y sus familiares de ser requerido. Finalmente, al término de la encuesta se solicitó una retroalimentación con el fin de mejorar la calidad de la entrevista.

\section{Análisis de datos y ética}

Se realizo una crítica a todas las fichas de recolección de datos aplicadas a cada paciente de la muestra de estudio para revisar que hayan sido llenadas correctamente, de encontrar fichas mal llenadas éstas fueron dejadas de lado. 
Una vez obtenido los datos, éstas se vaciaron digitalmente en el programa Microsoft Excel; el que además nos sirvió para la limpieza de la base de datos. Para un mejor análisis e interpretación de los datos obtenidos se utilizaron gráficos y tablas de frecuencias relativas y absolutas.

Asimismo, la presente investigación se realizó bajo estricto cumplimiento de los principios bioéticos, no se afectó ninguna norma de privacidad, ni los derechos a las personas encuestadas. Cada encuesta conto con el consentimiento informado firmado por el paciente, protegiendo su anonimato y respetando la confidencialidad de los datos brindados.

\section{RESULTADOS}

Se encuesto a 83 pacientes, de los cuales el 53\% fueron mujeres, con una media de edades de 54,5, siendo los adultos mayores en mayor proporción con un $39,8 \%$, su IMC tuvo como promedio 25,8 y un ICC que afecto fundamentalmente al sexo femenino en un 54\%. Respecto a los antecedentes familiares de riesgo cardiovascular la enfermedad con mayor prevalencia fue el infarto agudo de miocardio con un 38,5\%, en cuanto a los factores de riesgo cardiovascular personales, la diabetes mellitus fue la enfermedad con mayor prevalencia con un $65,6 \%$. Los datos están detallados en la Tabla 1.

Tabla 1. Características sociales y antropométricas en pacientes hospitalizados en un hospital de Lima.

\begin{tabular}{lcc}
\hline \multicolumn{1}{c}{ Sexo } & $\mathbf{N}^{\circ}$ & $\%$ \\
\hline Femenino & 44 & 53 \\
Masculino & 39 & 47 \\
EDAD* & 54,5 & 15 \\
Etapa de la vida Adulto & & \\
Adulto joven (20-24) & 7 & 8,4 \\
Adulto intermedio (25-54) & 30 & 36,1 \\
Adulto pre mayor (55-59) & 13 & 15,7 \\
Adulto mayor (más de 60 años) & 33 & 39,8 \\
Índice de masa corporal (IMC)* & 25,8 & 6,1 \\
Índice cintura cadera (ICC) & & \\
Varones & & 21 \\
Muy bajo (< 0.95) & 17 & 4,8 \\
Bajo (0.96-0.99) & 4 & 21 \\
Alto (>1.00) & 17 & 54 \\
Mujeres & & 26.3 \\
Alto (> 0.85) & 45 & 10,5 \\
Antecedente de Enfermedad cardiovascular familiar & & 38,5 \\
Infarto agudo de miocardio (IMA) & 10 & 34,6 \\
Hipertensión arterial (HTA) & 9 & 11,5 \\
Accidente cerebro vascular isquémico & 3 & \\
Antecedente de Enfermedad cardiovascular personal & & \\
Insuficiencia cardiaca congestiva (ICC) & 5 & \\
Infarto agudo de miocardio (IMA) & 4 & \\
Accidente cerebro vascular isquémico & 21 & \\
Diabetes mellitus tipo II & & \\
Dislipidemia (Colesterolemia) & & \\
\hline & & \\
\hline
\end{tabular}

*Media, Desviación Estándar.

Fuente: Elaboración propia. 
Al evaluar el régimen alimentario con mayor prevalencia, se observa que se consumen carnes blancas con mayor frecuencia $y$, al ser evaluados por la ansiedad, el 54,2\% sufren de ansiedad menor. Los resultados se hallan detallados en la Tabla 2.

Tabla 2. Régimen alimentario y grado de ansiedad en pacientes hospitalizados en un hospital de Lima.

\begin{tabular}{|c|c|c|}
\hline Régimen alimentario con mayor prevalencia & $\mathbf{N}^{\circ}$ & $\%$ \\
\hline Carbohidratos (Diario) & 47 & 56,6 \\
\hline Verduras (Diario) & 31 & 37,3 \\
\hline Frutas ( 2 a $3 /$ semana) & 27 & 32,5 \\
\hline Leche y derivados lácteos (1 vez por semana) & 21 & 25,3 \\
\hline Carnes rojas (no consume) & 33 & 39,8 \\
\hline Carnes blancas ( 2 a 3/semana) & 36 & 43,4 \\
\hline Lípidos (1 vez / semana) & 27 & 32,5 \\
\hline Comida Rápida (1 vez / semana) & 37 & 44,6 \\
\hline Test de Hamilton (Ansiedad) & $\mathbf{N}^{\circ}$ & $\%$ \\
\hline Sin ansiedad & 7 & 8,4 \\
\hline Ansiedad Menor & 45 & 54,2 \\
\hline Ansiedad clínicamente manifiesta & 4 & 4,8 \\
\hline Ansiedad Mayor & 27 & 32,5 \\
\hline
\end{tabular}

Fuente: Elaboración propia.

Con respecto a padecer de hipertensión arterial, se observa que el 21,7\% padecen de hipertensión, de los cuales el $73,9 \%$ están controlados y al comparar a aquellos pacientes que padecen de hipertensión arterial a la escala de hipertensión que nos da la Sociedad Europea de Cardiológica se tiene que 34,8 padecen de una hipertensión alta. Los resultados se hallan detallados en la Tabla 3.

Tabla 3. Hipertensión arterial en pacientes hospitalizados en un hospital de Lima.

\begin{tabular}{lcc}
\multicolumn{1}{c}{ Presión arterial } & $\mathbf{N}^{\circ}$ & $\%$ \\
Antecedente de hipertensión & 23 & 21,7 \\
Controladas & 17 & 73,9 \\
No controladas & 6 & 26,1 \\
Según la Sociedad Europea de Cardiología & & \\
Óptima & 5 & 21,7 \\
Normal 1 & 1 & 4.3 \\
Normal Alta & 8 & 34.8 \\
Hipertensión 1 & 5 & 21.7 \\
Hipertensión 2 & 1 & 4.3 \\
Hipertensión 3 & 0 & 0.0 \\
Hipertensión sistólica aislada & 3 & 13.0 \\
\hline
\end{tabular}

Fuente: Elaboración propia.

\section{DISCUSIÓN}

El aumento del IMC tiene una estrecha asociación con el riesgo de $\mathrm{ECV}^{15}$, sin embargo, se ha planteado que la distribución corporal del tejido adiposo es más importante que el peso corporal total para la determinación del riesgo cardiovascular $(\mathrm{CV})^{16}$, por tanto, existe un mayor interés en las mediciones antropométricas del riesgo y a una distribución más precisa de la masa adiposa y la masa magra por ello, la circunferencia de la cintura es un indicador de tejido adiposo en cintura y en el área abdominal y la circunferencia de cadera es un indicador del tejido adiposo que esta sobre los glúteos, es decir es un buen indicador para la grasa intrabdominal y visceral ${ }^{17}$, esto 
fue demostrado en estudios prospectivos mostrando una evidencia de asociación entre la adiposidad abdominal y la enfermedad coronaria a diferencia del IMC, en nuestro estudio se evidencio mayor ICC en mujeres, que en varones ${ }^{18-20}$, por tanto se podría pensar en un mayor riesgo de enfermedad coronaria.

La hipertensión arterial tiene gran prevalencia en América Latina y el Caribe ${ }^{21,22}$ pues el tener la presión elevada es un importante factor de riesgo de enfermedad coronaria, insuficiencia cardiaca, enfermedad cerebro vascular, insuficiencia renal y más recientemente fibrilación auricular ${ }^{23}$. Un elemento primordial en la prevención y rehabilitación es el ejercicio aeróbico con efectos antitrombóticos que reducen el riesgo de oclusión coronaria tras la rotura de una placa vulnerable, entre ellos, un aumento del volumen plasmático, una reducción de la viscosidad de la sangre, reducción de la agregación plaquetaria y una mayor capacidad trombolítica ${ }^{24}$, incluso el ejercicio reduce el riesgo de arritmias por la modulación favorable del balance autónomo ${ }^{25}$ La cantidad de actividad física/ejercicio aeróbico de intensidad moderada capaz de producir una reducción en la mortalidad CV y por todas las causas es de 2,5$5 \mathrm{~h} /$ semana $^{25}$. La historia familiar es una combinación variable de factores genéticos y factores ambientales compartidos, la evidencia indica que existe una alta probabilidad de heredar factores de riesgo como la diabetes mellitus ${ }^{26}$, refiriéndose que un control adecuado de la presión arterial en la diabetes mellitus reduciría el riesgo de eventos macro vasculares y micro vasculares, por tanto existe una relación positiva entre cifras de glicemia elevadas con mayor prevalencia de eventos cardiovasculares ${ }^{27}$.

La nutrición y calidad de alimentos influyen también a nivel cardiovascular particularmente en dislipidemias, la hipertensión arterial, obesidad y la diabetes; el efecto protector de las frutas y verduras parece ser más favorable mejorando de la presión arterial y la función microvascular, mientras que las asociaciones con las concentraciones de lípidos en plasma, el riesgo de DM y el peso corporal aún no se han establecido firmemente ${ }^{28}$ contrariamente, el consumo de zumos de fruta no aporta beneficio significativo además la fruta congelada o envasada aumentaría el riesgo de muerte un $17 \%$ por porción pero se debe considerar que algunas zonas del país tienen un acceso escaso a las frutas y verduras frescas afectando a las personas que tienen trastornos de salud preexistentes o estilos de vida complicados en aquellas que viven en zonas altoandinas.

Por último, la ansiedad generalizada aparece estar asociada con un elevado riesgo de enfermedad coronaria, incluso el elevado nivel de ansiedad estaría relacionado como un fuerte predictor de eventos fatales y no fatales de coronariopatías ${ }^{29}$. Las personas con ansiedad tienen un riesgo $26 \%$ superior de padecer enfermedades cardiovasculares y un $48 \%$ de muerte cardiovascular ${ }^{30}$.

\section{CONCLUSIÓN}

Los eventos con mayor frecuencia en la población estudiada fueron el ICC la diabetes, el antecedente familiar, el sedentarismo, el sobrepeso, la hipertensión no controlada, la dislipidemia, la dieta y el grado de ansiedad. Se recomienda estudios prospectivos y con mayor población con el fin de medir la fuerza de asociación de los elementos encontrados.

Contribuciones de autoría: Zuni-Chavez K, More Sandoval BD, Fernández-Vargas CD, García-Fuentes BB, Ruiz-Olano JM, Perez-Rodriguez VK; contribuyeron en redacción y revisión final del artículo de investigación, Zuni-Chavez KX, contribuyó en la revisión final del artículo.

Financiamiento: Autofinanciado.

Conflicto de interés: Los autores declaran no tener conflicto de interés en la publicación de este artículo.

Recibido: 30 de mayo del 2019

Aprobado: 05 de setiembre del 2019

Correspondencia: Karla X. Zuni-Chavez

Dirección: Avenida Colombia 247, Lima 21, Perú.

Teléfono: 979552526

Correo:kxiomara23@hotmail.com 


\section{REFERENCIAS BIBLIOGRÁFICAS}

1.Montanaro C. Cardiovascular risk in adolescents Int J Cardiol. 2017;240:444-5. Disponible en: https://www. internationaljournalofcardiology.com/article/S0167-5273(17)31505-X/ fulltext

2.Paynter NP. Cardiovascular Risk Prediction: Widening the Net. Circ Res. 2017;121(9):1032-3.Dosponible en: https://www.ahajournals.org/ doi/full/10.1161/CIRCRESAHA.117.311868?url_ver=Z39.88-2003\&rfr_ id=ori\%3Arid\%3Acrossref.org\&rfr_dat=cr_pub\%3Dpubmed

3.Martínez-Espichán $\mathrm{Y}$, Zambrano-Huailla $\mathrm{R}$, Zambrano-Huailla A, Manrique-Acha A, Mayta-Calderón JC, Cardenas-Rojas A, et al. Características de los factores de riesgo cardiovascular en una población urbana y rural de la selva peruana, julio - 2014. Horiz Méd Lima. abril de 2017;17(2):38-42. Disponible en: http://www.horizontemedicina.usmp. edu.pe/index.php/horizontemed/article/view/579

4.Karmali KN, Lloyd-Jones DM. Implementing Cardiovascular Risk Prediction in Clinical Practice: The Future Is Now. J Am Heart Assoc. 24 de 2017;6(4). Dsiponible en: https://www.ahajournals.org/doi/full/10.1161/ JAHA.117.006019?url_ver=Z39.88-2003\&rfr_id=ori:rid:crossref.org\&rfr dat $=$ cr_pub\%3dpubmed

5.Graham IM. The importance of total cardiovascular risk assessment in clinical practice. Eur J Gen Pract. 2006;12(4):148-55. Disponible en https://www.tandfonline.com/doi/full/10.1080/13814780600976282

6.Enfermedades cardiovasculares [Internet]. Organizacion Mundial de la Salud. 2017. Disponible en: https://www.who.int/es/news-room/factsheets/detail/cardiovascular-diseases-(cvds).

7.Van den Brekel-Dijkstra K, Rengers AH, Niessen MAJ, de Wit NJ, Kraaijenhagen RA. Personalized prevention approach with use of web-based cardiovascular risk assessment with tailored lifestyle followup in primary care practice--a pilot study. Eur J Prev Cardiol. marzo de 2016;23(5):544-51. Disponible en: https://journals.sagepub.com/doi/ abs/10.1177/2047487315591441?rfr_dat=cr_pub\%3Dpubmed\&url ver=Z39.88-2003\&rfr_id=ori\%3Arid\%3Acrossref.org\&journalCode=cprc

8.Ramôa Castro A, Oliveira NL, Ribeiro F, Oliveira J. Impact of educational interventions on primary prevention of cardiovascular disease: $A$ systematic review with a focus on physical activity. Eur J Gen Pract. diciembre de 2017;23(1):59-68. Disponible en: https://www.tandfonline. com/doi/full/10.1080/13814788.2017.1284791

9.González-López JJ, Gómez-Arnau Ramírez J, Torremocha García R Albelda Esteban S, Alió del Barrio J, Rodríguez-Artalejo F. Conocimientos sobre los procedimientos correctos de medición de la presión arterial entre estudiantes universitarios de ciencias de la salud. Rev Esp Cardiol. de mayo de 2009;62(5):568-71.Disponible en: https://www.revespcardiol. org/es-conocimientos-sobre-los-procedimientos-correctosarticulo-13136003

10.Tagle R. DIAGNÓSTICO DE HIPERTENSIÓN ARTERIAL. Rev Médica Clínica Las Condes. 1 de enero de 2018;29(1):12-20. Disponible en https://www.elsevier.es/es-revista-revista-medica-clinica-las-condes 202-articulo-diagnostico-de-hipertension-arterial-S0716864018300099

11.Perk J, De Backer G, Gohlke H, Graham I Reiner Z, Verschuren M, et al. European Guidelines on cardiovascular disease prevention in clinica practice (version 2012). The Fifth Joint Task Force of the European Society of Cardiology and Other Societies on Cardiovascular Disease Prevention in Clinical Practice (constituted by representatives of nine societies and by invited experts). Eur Heart J. julio de 2012;33(13):1635-701. Disponible en: https://academic.oup.com/eurheartj/article/33/13/1635/488083

12.Instituto Nacional de Salud. Centro Nacional de Alimentacion y Nutricion [Internet]. INSTITUTO NACIONAL DE SALUD. 2019 [citado 15 de septiembre de 2019]. Disponible en: http://web.ins.gob.pe/es/ alimentacion-y-nutricion/acerca-de-cenan/presentacion

13. Hamilton $\mathrm{M}$. The assessment of anxiety states by rating. $\mathrm{Br} \mathrm{J}$ Med Psychol. 1959;32(1):50-5. Disponible en: https://onlinelibrary.wiley.com/ doi/10.1111/j.2044-8341.1959.tb00467.x

14.Thompson E. Hamilton Rating Scale for Anxiety (HAM-A). Occup Med Oxf Engl. octubre de 2015;65(7):601. Disponible en: https://academic oup.com/occmed/article/65/7/601/1733495

15.López-Jiménez F, Cortés-Bergoderi M. Obesidad y corazón. Rev Esp Cardiol. 2011;64(2):140-9. Dlsponible en: https://www.revespcardiol.org/ es-obesidad-corazon-articulo-S0300893210000667
16.Bryce-Moncloa A, Alegría-Valdivia E, San Martin-San Martin MG. Obesidad y riesgo de enfermedad cardiovascular. An Fac Med. abril de 2017;78(2):202-6. Disponible en: http://www.scielo.org.pe/scielo. php?script=sci_arttext\&pid=S1025-55832017000200016

17.Pérez León S, Díaz-Perera Fernández G. Circunferencia de la cintura en adultos, indicador de riesgo de aterosclerosis. Rev Habanera Cienc Médicas. diciembre de 2011;10(4):441-7. Disponible en: http://scielo.sld. cu/scielo.php?script=sci_arttext\&pid=S1729-519X2011000400005

18.Romero-Velarde E, Vásquez-Garibay EM, Álvarez-Román YA, FonsecaReyes S, Casillas Toral E, Troyo Sanromán R. Circunferencia de cintura y su asociación con factores de riesgo cardiovascular en niños y adolescentes con obesidad. Bol Méd Hosp Infant México. octubre de 2013;70(5):35863. Disponible en: http://www.scielo.org.mx/scielo.php?script=sci_ arttext\&pid=S1665-11462013000500004

19.González M Ignacio M. Circunferencia de cintura: una medición importante y útil del riesgo cardiometabólico. Rev Chil Cardiol. 2010;29(1):85-7. Disponible en: https://scielo.conicyt.cl/scielo. php?script=sci arttext\&pid=S0718-85602010000100008

20.Dávila-Sotelo E, Flores-Caloca Ó, Cura-Esquivel I, Caballero-Talavera T, Estrada-Zúñiga C, de la O-Cavazos ME, et al. Correlación de circunferencia de cintura con factores de riesgo cardiovascular en niños. Med Univ. 1 de octubre de 2012;14(57):211-6. Disponible en: https://www.elsevier.es/esrevista-medicina-universitaria-304-articulo-correlacion-circunferenciacintura-con-factores-X1665579612844278

21.Ruilope LM, Chagas ACP, Brandão AA, Gómez-Berroterán R, Alcalá $J J A$, Paris JV, et al. Hypertension in Latin America: Current perspectives on trends and characteristics. Hipertens Riesgo Vasc. 1 de enero de 2017;34(1):50-6. Disponible en: https://www.sciencedirect.com/science/ article/abs/pii/S1889183716300605

22.Revisión sistemática de literatura: Determinantes sociales de la salud en hipertensión, América Latina 2006-2014. | Salutem Scientia Spiritus. [citado 15 de septiembre de 2019]; Disponible en: https:// revistas.javerianacali.edu.co/index.php/salutemscientiaspiritus/article/ view/1694

23.Arredondo A, Zuñiga A. Cambios epidemiológicos y consecuencias financieras de la hipertensión en América Latina: implicaciones para el sistema de salud y los pacientes en México. Cad Saúde Pública. marzo de 2012;28(3):497-502.Disponible en: http://www.scielo.br/scielo. php?pid=S0102-311X2012000300010\&script=sci_abstract\&tlng=es

24.García Delgado JA, Pérez Coronel PL, Chí Arcia J, Martínez Torrez J, Pedroso Morales I. Efectos terapéuticos del ejercicio físico en la hipertensión arterial. Rev Cuba Med. septiembre de 2008;47(3):00 . Disponible en: http://scielo.sld.cu/scielo.php?script=sci arttext\&pid=S0034-75232008000300002

25.Segura J, Vinyoles E. El efecto del ejercicio físico sobre la presión arteria a partir de los 55 años. Hipertens Riesgo Vasc. enero de 2005;22(6):269-70. Disponible en: https://www.elsevier.es/es-revista-hipertension-riesgovascular-67-pdf-S1889183705715634

26.Mazón-Ramos P, Cordero A, González-Juanatey JR, Bertomeu Martínez V, Delgado E, Vitale G, et al. Control de factores de riesgo cardiovascular en pacientes diabéticos revascularizados: un subanálisis del estudio ICPBypass. Rev Esp Cardiol. 1 de febrero de 2015;68(2):115-20. Disponible en: https://www.revespcardiol.org/es-control-factores-riesgocardiovascular-pacientes-articulo-S0300893214003200? redirect=true

27.Gaede $\mathrm{P}$, Vedel $\mathrm{P}$, Parving $\mathrm{HH}$, Pedersen $\mathrm{O}$. Intensified multifactoria intervention in patients with type 2 diabetes mellitus and microalbuminuria: the Steno type 2 randomised study. Lancet Lond Engl. 20 de febrero de 1999;353(9153):617-22. Disponible en: https://www. thelancet.com/journals/lancet/article/PIIS0140-6736(98)07368-1/fulltext

28.Ortega Anta RM, Jiménez Ortega Al, Perea Sánchez JM, Cuadrado Soto E, López-Sobaler AM. Pautas nutricionales en prevención y control de la hipertensión arterial. Nutr Hosp. 2016:33:53-8. Disponible en: http://scielo. isciii.es/scielo.php?script=sci_abstract\&pid=S0212-16112016001000013

29.Valero Zanuy MÁ. Nutrición e hipertensión arterial. Hipertens Riesgo Vasc. 1 de enero de 2013;30(1):18-25. Disponible en: https://medes.com/ publication/79945

30.Molerio Pérez O, García Romagosa G. Influencia del estrés y las emociones en la hipertensión arterial esencial. Rev Cuba Med. junio de 2004;43(2-3):0-0. Disponible en: http://scielo.sld.cu/scielo. php?script=sci_arttext\&pid=S0034-75232004000200007 